Pacific

Journal of

Mathematics

THE MINIMAL HIRSCH-BROWN MODEL VIA CLASSICAL HODGE THEORY

Christopher Allday And Volker Puppe 


\title{
THE MINIMAL HIRSCH-BROWN MODEL VIA CLASSICAL HODGE THEORY
}

\author{
Christopher Allday AND Volker Puppe
}

\begin{abstract}
In our book on cohomological methods in transformation groups the minimal Hirsch-Brown model was used to good effect. The construction of the model there, however, was rather abstract. Here, for smooth compact connected Lie group actions on smooth closed manifolds, we give a much more explicit construction of the minimal Hirsch-Brown model using operators from classical Hodge theory and the Cartan model.
\end{abstract}

\section{Introduction}

The minimal Hirsch-Brown model is described in detail and used to good effect in [Allday and Puppe 1993, $\S 1.3,1.4,4.4,4.6]$. The construction of the minimal Hirsch-Brown model there, however, is rather abstract. Our purpose here is to give a more explicit construction of the model for smooth compact connected Lie group actions on closed smooth manifolds using operators from classical Hodge theory. Two of our main results, Theorem 3.7 and Corollary 3.8, are particularly nice in view of their relation to [Alekseev and Meinrenken 2005].

In Section 2 we introduce our notation, and describe how Hodge theory applies to the Cartan model for computing equivariant cohomology. Section 3 gives the explicit construction of the minimal Hirsch-Brown model. Section 4, as an example, discusses the familiar product structure in the equivariant cohomology of a Hamiltonian circle action on $\mathbb{C} P^{n}$. We compute the deformation of the product (going from ordinary to equivariant cohomology) in terms of the moment map in two different ways.

\section{Notation and review}

Let $G$ be a compact connected Lie group acting smoothly on a closed smooth manifold $M$. Suppose that $M$ has an invariant Riemannian metric $r$. If one does Hodge theory with respect to $r$, then all the usual operators, for example, $*, d^{*}$, the projection onto the harmonic forms $\pi \mathscr{H}$, the Laplacian $\Delta$, and Green's operator

MSC2000: primary 57S15, 57R91; secondary 57R17, 53D05.

Keywords: Cartan model, Hirsch-Brown model, Hodge theory, canonical extension. 
$\mathscr{G}$ are invariant. Since the Lie group $G$ is connected, it acts trivially on the cohomology of $M$, so all harmonic forms are invariant. Let $\Omega_{i n v}(M)$ or $\Omega(M)^{G}$ denote the cochain complex of invariant (i.e., fixed) forms. For $\alpha \in \Omega(M)$, the standard Hodge decomposition is

$$
\alpha=\pi_{\mathscr{H}}(\alpha)+\Delta \mathscr{G}(\alpha)=\pi_{\mathscr{H}}(\alpha)+d d^{*} \mathscr{G}(\alpha)+d^{*} d^{\mathscr{G}}(\alpha) .
$$

Since we are using an invariant Riemannian metric, if $\alpha \in \Omega_{\text {inv }}(M)$, then each of $\pi_{\mathscr{H}}(\alpha), d d^{*} \mathscr{G}(\alpha)$, and $d^{*} d \mathscr{G}(\alpha)$ is in $\Omega_{\text {inv }}(M)$. For an introduction to Hodge theory, see [Warner 1983].

In this paper we shall always assume that the Lie group $G$, the manifold $M$, and the Riemannian metric are as in the paragraph above.

We shall construct the Hirsch-Brown model from the Cartan model for computing equivariant cohomology $H_{G}^{*}(M ; \mathbb{R})$. Recall that the Cartan model, denoted $C_{G}(M)$, is

$$
\left(S\left(\mathfrak{g}^{*}\right) \otimes \Omega(M)\right)^{G}
$$

with differential $d_{G}=d-\partial$. Here, $\partial=\sum_{j=1}^{n} t_{j} \otimes i_{j}$, where $n=\operatorname{dim}(G)$, the $t_{j}$ form a basis for the dual $\mathfrak{g}^{*}$ of the Lie algebra of $G$, and $i_{j}$ for $1 \leq j \leq n$ is the inner product with the vector field induced by the dual basis element of $\mathfrak{g}$ corresponding to $t_{j}$. (See [Guillemin and Sternberg 1999, §4.2].)

In the paragraph above, we abbreviated $I \otimes d$ by $d$. Throughout this paper we shall abbreviate operators such as $I \otimes \pi_{\mathscr{H}}, I \otimes d^{*}, I \otimes \Delta$ and $I \otimes \mathscr{G}$ on $C_{G}(M)$ simply as $\pi_{\mathscr{H}}, d^{*}, \Delta$ and $\mathscr{G}$.

We shall also let $R_{G}=S\left(\mathfrak{g}^{*}\right)^{G} \cong H^{*}(B G ; \mathbb{R})$, and let $\mathscr{H} \subseteq \Omega_{\text {inv }}(M)$ denote the subspace of harmonic forms.

Lemma 2.1. In $C_{G}(M)$, there is the Hodge decomposition

$$
\begin{aligned}
C_{G}(M) & =\left(S\left(\mathfrak{g}^{*}\right) \otimes \mathscr{H}\right)^{G} \oplus\left(S\left(\mathfrak{g}^{*}\right) \otimes d \Omega(M)\right)^{G} \oplus\left(S\left(\mathfrak{g}^{*}\right) \otimes d^{*} \Omega(M)\right)^{G} \\
& =R_{G} \otimes \mathscr{H} \oplus d C_{G}(M) \oplus d^{*} C_{G}(M) .
\end{aligned}
$$

Proof. By the standard Hodge decomposition, $\Omega(M)=\mathscr{H} \oplus d \Omega(M) \oplus d^{*} \Omega(M)$. So the first form of the Hodge decomposition of $C_{G}(M)$ follows since $\mathscr{H}, d \Omega(M)$ and $d^{*} \Omega(M)$ are $G$-invariant. As $\mathscr{H} \subseteq \Omega(M)^{G}$, it follows that $\left(S\left(\mathfrak{g}^{*}\right) \otimes \mathscr{H}\right)^{G}=R_{G} \otimes \mathscr{H}$.

Now let $S\left(\mathfrak{g}^{*}\right) \otimes \Omega(M)=C$ for brevity. Since $d$ and $d^{*}$ are $G$-equivariant, they preserve the decomposition of $C$ into the kernel and image of the $G$-averaging operator. Hence $(d C)^{G}=d C^{G}=d C_{G}(M)$, and similarly for $d^{*}$.

Next we define two operators on $C_{G}(M)$ which play an important role in our description of the minimal Hirsch-Brown model and its relation to the Cartan model.

Definition 2.2. On $C_{G}(M)$, let $P=d^{*} \mathscr{G}$ and $Q=\partial d^{*} \mathscr{G}$. 
Remarks 2.3. (1) Since $d^{*}$ and $\varphi$ commute, $P Q=Q P=0$.

(2) Since $P$ and $Q$ lower degrees in $\Omega(M)$, the operators $I-P$ and $I-Q$ are invertible.

\section{The minimal Hirsch-Brown model}

We now consider the commutative diagram given by restricting $I-Q$ to $d C_{G}(M)$. Lemma 3.1. The following diagram commutes, where the top arrow is the inclusion.

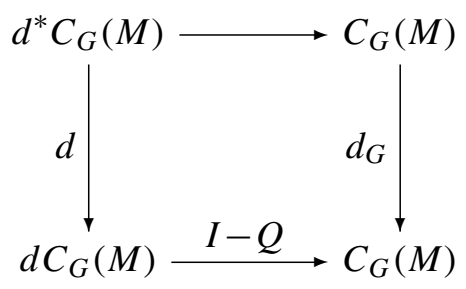

To put it another way, $d_{G} d^{*} G\left|d C_{G}(M)=(I-Q)\right| d C_{G}(M)$.

Proof. $(I-Q) d\left|d^{*} C_{G}(M)=\left(d-\partial d^{*} d \varphi\right)\right| d^{*} C_{G}(M)=d_{G} \mid d^{*} C_{G}(M)$, because $d^{*} d \varphi$ is the identity on the image of $d^{*}$.

Definition 3.2. (1) On $C_{G}(M)$, set $D=(I-Q)^{-1} d_{G}(I-Q)$.

(2) On $R_{G} \otimes \mathscr{H}$, define the Hirsch-Brown differential $d_{H B}=\pi_{\mathscr{H}} D \mid R_{G} \otimes \mathscr{H}$.

It is clear that $D^{2}=0$. That $d_{H B}^{2}=0$ follows from

Lemma 3.3. The following diagram commutes.

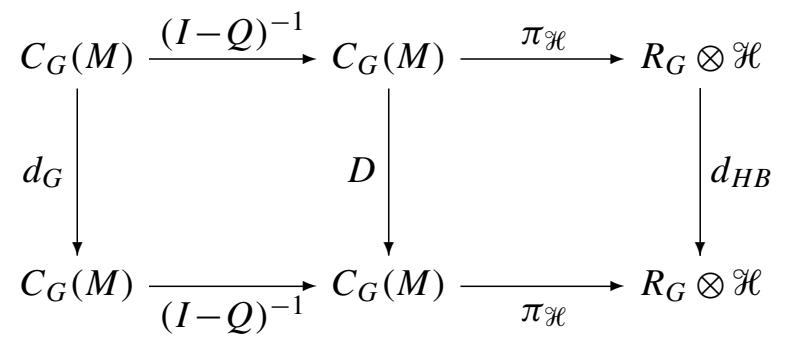

Proof. It is enough to show that $\pi_{\mathscr{H}} D(\alpha)=0$ for any $\alpha \in d \Omega(M) \oplus d^{*} \Omega(M)$. This follows from Lemma 3.4.

Lemma 3.4. (1) On $R_{G} \otimes \mathscr{H}, D=-(I-Q)^{-1} \partial=-\partial(I-P)^{-1}$.

(2) $\operatorname{Ond}_{G}(M), D=0$.

(3) $O n d^{*} C_{G}(M), D=d$.

Proof. (1) On $R_{G} \otimes \mathscr{H}$, both $d=0$ and $Q=0$. So

$$
D=(I-Q)^{-1} d_{G}(I-Q)=(I-Q)^{-1} d_{G}=-(I-Q)^{-1} \partial .
$$

This part then follows since $Q \partial=\partial P$. 
(2) From Lemma 3.1, $(I-Q)\left|d C_{G}(M)=d_{G} d^{*} \varphi\right| d C_{G}(M)$. Hence we have $d_{G}(I-Q) \mid d C_{G}(M)=0$.

(3) On $d^{*} C_{G}(M)$, we have $Q=0$. Thus $D=(I-Q)^{-1} d_{G}=(I-Q)^{-1}((I-Q) d)$ on $d^{*} C_{G}(M)$ by Lemma 3.1.

Definition 3.5. The minimal Hirsch-Brown model for $H_{G}^{*}(M ; \mathbb{R})$ is the differential $R_{G}$-module $\left(R_{G} \otimes \mathscr{H}, d_{H B}\right)=\left(H^{*}(B G ; \mathbb{R}) \otimes H^{*}(M ; \mathbb{R}), d_{H B}\right)$.

Lemma 3.6. $H\left(R_{G} \otimes \mathscr{H}, d_{H B}\right) \cong H_{G}^{*}(M ; \mathbb{R})$, where the $H$ on the left means (co)homology with respect to the differential $d_{H B}$.

Proof. Since $I-Q$ is an isomorphism, $(I-Q)^{-1}$ induces an isomorphism on cohomology. By Lemma 3.4 (2) and (3), $d C_{G}(M) \oplus d^{*} C_{G}(M)=\operatorname{ker} \pi \mathscr{H}$ is acyclic with respect to $D$. So $\pi_{\mathscr{H}}$ also induces an isomorphism in cohomology.

The Hirsch-Brown differential can be written in a very useful way:

Theorem 3.7. $O n R_{G} \otimes \mathscr{H}$,

$$
d_{H B}=(I-P) d_{G}(I-P)^{-1} .
$$

Proof. Let $a \in R_{G} \otimes \mathscr{H}$. By Lemma 3.4(1) and the Hodge Decomposition Theorem,

$$
\begin{aligned}
d_{H B}(a) & =\pi_{\mathscr{H}} D(a)=-\pi_{\mathscr{H}} \partial(I-P)^{-1}(a) \\
& =-\partial(I-P)^{-1}(a)+\Delta \varphi \partial(I-P)^{-1}(a) .
\end{aligned}
$$

In general, though, $\Delta \mathscr{G} \partial=d^{*} \mathscr{G} d \partial+d d^{*} \varphi \partial=d P-P d$, that is, $[d, P]=\Delta \mathscr{G} \partial$. So

$$
\begin{aligned}
\Delta^{\varphi} \varphi(I-P)^{-1}(a) & =d P(I-P)^{-1}(a)-P d(I-P)^{-1}(a) \\
& =d(I-(I-P))(I-P)^{-1}(a)-P d(I-P)^{-1}(a) \\
& =d(I-P)^{-1}(a)-P d(I-P)^{-1}(a),
\end{aligned}
$$

where the last equality follows since $d(a)=0$. Finally, as $P \partial=0$, we have

$$
d_{H B}(a)=-\partial(I-P)^{-1}(a)+(I-P) d(I-P)^{-1}(a)=(I-P) d_{G}(I-P)^{-1}(a) .
$$

Corollary 3.8. The following diagram commutes, where $i_{\mathscr{H}}$ is the inclusion.

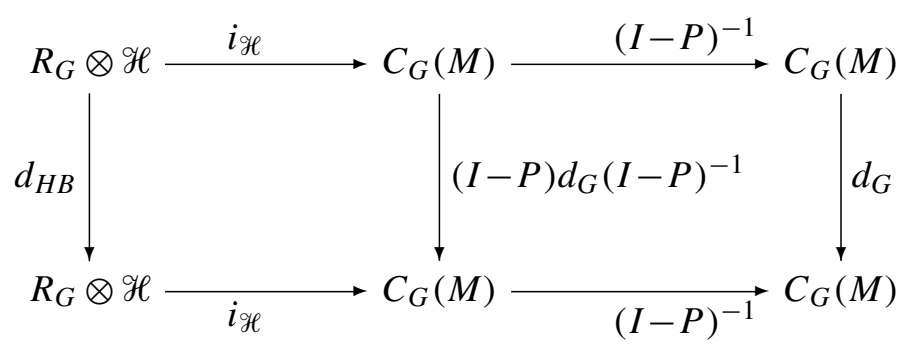


Furthermore, $\pi_{\mathscr{H}}(I-Q)^{-1}(I-P)^{-1} i_{\mathscr{H}}=I$.

Proof. Since both $\pi_{\mathscr{H}} P=0$ and $Q i_{\mathscr{H}}=0$, we have $\pi_{\mathscr{H}}(I-P)^{-1}=\pi_{\mathscr{H}}$ and $(I-Q)^{-1} i_{\mathscr{H}}=i_{\mathscr{H}}$. Also $P$ and $Q$ commute.

So $\pi_{\mathscr{H}}(I-Q)^{-1}(I-P)^{-1} i_{\mathscr{H}}=\pi_{\mathscr{H}}(I-P)^{-1}(I-Q)^{-1} i_{\mathscr{H}}=\pi_{\mathscr{H}} i_{\mathscr{H}}=I$.

Remark 3.9. Corollary 3.8 shows that $\pi_{\mathscr{L}}(I-Q)^{-1}$ is a fibration of differential $R_{G}$-modules in the sense of [Allday and Puppe 1993, Definition B.1.5]. It follows that $\pi_{\mathscr{H}}(I-Q)^{-1}$ is a homotopy equivalence of differential $R_{G}$-modules by [Allday and Puppe 1993, Propositions B.1.8, B.1.9].

Definition 3.10. As in [Allday 2005], we use the letters CEF to abbreviate "cohomology extension of the fibre", meaning that the map

$$
i^{*}: H_{G}^{*}(M ; \mathbb{R}) \rightarrow H^{*}(M ; \mathbb{R})
$$

is surjective, where $i: M \rightarrow M_{G}$ is the inclusion of the fibre in the Borel construction bundle $M_{G} \rightarrow B G$.

The term "cohomology extension of the fibre" is traditional [Spanier 1966]. Our use of CEF here is intended as a compromise between the term "totally nonhomologous to zero" (TNHZ), which has been used in the cohomology theory of transformation groups for a very long time, and the more recent term "equivariantly formal", which, we feel, should mean more than just CEF. (See [Lillywhite 2003].)

Theorem 3.7 reproves the main result of [Allday 2005], as we show next.

Corollary 3.11. Let $G$ be any compact connected Lie group and suppose that there is a CEF. Let $\alpha \in \Omega_{\text {inv }}(M)$ be a harmonic form. Then $(I-P)^{-1}(\alpha)$ is a canonical equivariant extension of $\alpha$. That is

$$
i^{*}(I-P)^{-1}(\alpha)=\alpha \quad \text { and } \quad d_{G}(I-P)^{-1}(\alpha)=0 .
$$

Proof. Since $i^{*}$ is surjective, it follows that

$$
H_{G}^{*}(M ; \mathbb{R}) \cong R_{G} \otimes H^{*}(M ; \mathbb{R})
$$

as a $R_{G}$-module. Hence $d_{H B}=0$. Thus by Theorem 3.7, $d_{G}(I-P)^{-1}(\alpha)=0$, for all $\alpha \in \mathscr{H}$.

The operators used above, $P, Q, \pi_{\mathscr{H}}, \Delta$ and $\mathscr{G}$, for example, are not multiplicative, so the product in the Cartan model does not carry over in a simple way to the minimal Hirsch-Brown model. We shall not discuss products in the Hirsch-Brown model in a general context in this paper. When a CEF exists however, $d_{H B}=0$, so

$$
R_{G} \otimes \mathscr{H} \cong H_{G}^{*}(M ; \mathbb{R}) .
$$

In this case, we shall take the product in $R_{G} \otimes \mathscr{H}$ to be the usual cup product in $H_{G}^{*}(M ; \mathbb{R})$ via the isomorphism above. 
When $G$ is the circle group $S^{1}$, and there is a CEF, then the cup product in $H_{G}^{*}(M ; \mathbb{R})$ is a deformation of the cup product in $H^{*}(M ; \mathbb{R})$. More generally, for compact connected $G$, when there is a CEF, the cup product in equivariant cohomology can be viewed as a deformation of the cup product in ordinary cohomology over the parameter space $R_{G}$.

Definition 3.12. (1) In the CEF case, let $\widetilde{\wedge}$ denote the product in the minimal Hirsch-Brown model. In particular, if $\alpha, \beta \in \mathscr{H} \subseteq \Omega_{\text {inv }}(M)$, then $\alpha \widetilde{\wedge} \beta$ is the product of $\alpha$ and $\beta$ in $R_{G} \otimes \mathscr{H}$, whereas of course, $\alpha \wedge \beta$ is the product in $\Omega_{\text {inv }}(M)$.

(2) For $\alpha \in \Omega(M)$, abbreviate $(I-P)^{-1}(\alpha)$ by $\hat{\alpha}$.

We now have the following description of the cup product in $H_{G}^{*}(M ; \mathbb{R})$ in the CEF case. Of course, as $R_{G}$-modules, $H_{G}^{*}(M ; \mathbb{R}) \cong R_{G} \otimes \mathcal{H}$. Since $H_{G}^{*}(M ; \mathbb{R})$ is a $R_{G}$-algebra, it is enough to describe $\widetilde{\wedge}$ on $\mathscr{H}$.

Proposition 3.13. Suppose that there is a CEF. Then, for $\alpha, \beta \in \mathscr{H}$,

$$
\alpha \widetilde{\wedge}=\pi_{\mathscr{H}}(1-Q)^{-1}(\hat{\alpha} \hat{\beta}) .
$$

Proof. Let $\theta=\pi_{\mathscr{H}}(1-Q)^{-1}$. Since $d_{H B}=0$, Corollary 3.11 implies that $\hat{\alpha}$ and $\hat{\beta}$ are cycles in $C_{G}(M)$. As the Cartan model is multiplicative, the product $\hat{\alpha} \hat{\beta}$ in $C_{G}(M)$ represents $[\hat{\alpha}][\hat{\beta}]$, the product in $H_{G}^{*}(M ; \mathbb{R})$. Although $\theta$ is not multiplicative, $\theta^{*}$ is an isomorphism, so we have

$$
\alpha \widetilde{\wedge} \beta=\theta^{*}([\hat{\alpha}]) \theta^{*}([\hat{\beta}])=\theta^{*}([\hat{\alpha}][\hat{\beta}])=\theta^{*}([\hat{\alpha} \hat{\beta}])=[\theta(\hat{\alpha} \hat{\beta})]=\theta(\hat{\alpha} \hat{\beta}),
$$

where the first and last equalities hold since $d_{H B}=0$.

Remarks 3.14. (1) Since $\hat{\alpha}=(I-P)^{-1}(\alpha)$, for $\alpha, \beta \in \mathscr{H}$, under the conditions of Proposition 3.13,

$$
\alpha \widetilde{\wedge} \beta=\pi_{\mathscr{H}}(\alpha \wedge \beta) \text { modulo } \bar{R}_{G} \otimes \mathscr{H},
$$

where $\bar{R}_{G}$ is the augmentation ideal of elements of positive degree in $R_{G}$ and $\pi_{\mathscr{H}}(\alpha \wedge \beta)$ is the product of $\alpha$ and $\beta$ in $\mathscr{H} \cong H^{*}(M ; \mathbb{R})$.

(2) Let a compact connected Lie group $G$ act on a closed manifold $M$, and let $T \subseteq G$ be a maximal torus with Weyl group $W$. Then there is a homomorphism of complexes $j_{T}: C_{G}(M) \rightarrow C_{T}(M)^{W}$ that induces an isomorphism in cohomology [Guillemin and Sternberg 1999, §6.8]. It is easy to see that $C_{T}(M)^{W}$ is a free $R_{G^{-}}$ module. Thus $j_{T}(I-P)^{-1} i_{\mathscr{H}}: R_{G} \otimes \mathscr{H} \rightarrow C_{T}(M)^{W}$ is a homotopy equivalence of differential $R_{G}$-modules [Allday and Puppe 1993, Remark B.1.10, Propositions B.1.11, B.1.7].

(3) If $(M, \omega)$ is a closed symplectic manifold and the action of a compact connected Lie group $G$ on $M$ is symplectic, then the action is Hamiltonian if and only if $[\omega] \in H^{*}(M ; \mathbb{R})$ is in the image of $i^{*}$. This follows directly from the Cartan model [Guillemin and Sternberg 1999, §9.1]. The latter holds for $G$ if and only if 
it holds for any maximal torus $T$ of $G$. By [Frankel 1959], if the action of $T$ is Hamiltonian, there is a CEF for every subcircle of $T$. By standard results from the cohomology theory of transformation groups, such as [Hsiang 1975] or [Allday and Puppe 1993, Theorem 3.10.4], if there is a CEF for every subcircle, then there is a CEF for $T$ (by choosing a subcircle $C \subseteq T$ such that $M^{C}=M^{T}$ ). And, by (2) above, there is a CEF for $G$ if and only if there is a CEF for $T$. So there is a CEF for $G$ if and only if the action is Hamiltonian. Before the Cartan model was well understood, this result was obtained by Kirwan [1984] using different methods.

(4) An argument very similar to the proof of Theorem 3.7 shows that for any $\alpha \in \Omega_{\text {inv }}(M)$,

$$
(I-P) d_{G}(I-P)^{-1}(\alpha)=\pi_{\mathscr{H}} d_{G}(I-P)^{-1}(\alpha)+d \alpha .
$$

(Briefly, $\pi_{\mathscr{H}} d_{G}(\hat{\alpha})=-\pi_{\mathscr{H}} \partial(\hat{\alpha})=-\partial(\hat{\alpha})+\Delta \varphi \partial(\hat{\alpha})=-\partial(\hat{\alpha})+d P(\hat{\alpha})-P d(\hat{\alpha})$

$$
\left.=-\partial(\hat{\alpha})+d(\hat{\alpha}-\alpha)-P d(\hat{\alpha})=d_{G} \hat{\alpha}-P d_{G}(\hat{\alpha})-d \alpha .\right)
$$

(5) Assuming that there is a CEF, from Corollary 3.8 it follows similarly that, for any $\alpha, \beta \in \mathscr{H}$, there is $\gamma \in C_{G}(M)$ such that

$$
(I-P)^{-1} i_{\mathscr{H}}(\alpha \widetilde{\wedge} \beta)=\hat{\alpha} \hat{\beta}+d_{G} \gamma .
$$

(6) Similar results hold for products of three or more elements.

\section{An example}

Let $M$ be a closed symplectic $2 n$-manifold with symplectic form $\omega$. Suppose that a compact connected Lie group $G$ is acting on $M$ in a Hamiltonian way. Then we may choose an invariant Riemannian metric on $M$ that is compatible with $\omega$ [McDuff and Salamon 1995, Lemma 5.49]. So, if $r$ is the metric, and $V_{1}$ and $V_{2}$ are any two vector fields on $M$, then $r\left(V_{1}, V_{2}\right)=\omega\left(V_{1}, J V_{2}\right)$, where $J$ is an invariant compatible almost-complex structure on $M$. It follows that $\omega^{j}$ is harmonic for $0 \leq j \leq n$, and

$$
*\left(\frac{\omega^{j}}{j !}\right)=\frac{\omega^{n-j}}{(n-j) !},
$$

for $0 \leq j \leq n$. In particular, $\omega^{n} / n$ ! is the volume form.

As remarked above, in the Hamiltonian case, $M$ has a CEF, so in the minimal Hirsch-Brown model, $d_{H B}=0$. Thus the remaining problem is to determine the product structure in $H_{G}^{*}(M ; \mathbb{R})$. In this section we shall do this in the familiar situation where $G=S^{1}$ and $M=\mathbb{C} P^{n}$. The results are not new, although they may be assembled in a somewhat novel way.

First, however, consider a Hamiltonian action of $G=S^{1}$ on any closed symplectic manifold $(M, \omega)$. Let $\mu$ be the moment map and suppose that $\mu$ has been chosen to have average value zero on $M$, that is, $\int_{M} \mu \omega^{n} / n !=0$. Let $V$ be the 
vector field defined by the circle action: for any $x \in M$,

$$
V_{x}=\left.\frac{d}{d u} \exp (2 \pi i u) x\right|_{u=0} .
$$

In the Cartan model then, the differential $d_{G}=d-t i_{V}$, where $t \in H^{2}(B G ; \mathbb{R})$ is the polynomial generator. In the Hodge decomposition $\mu=d^{*} d^{\mathscr{G}} \mu$, since the harmonic part $\pi \mathscr{H}(\mu)$ is the average value. Thus $P(\omega)=t \mu$, because $d^{*} \varphi_{i} i_{V}(\omega)=d^{*} \varphi_{d} d \mu=\mu$. Hence $\widehat{\omega}=\omega+t \mu$, the standard equivariant extension of $\omega$.

From now on, we denote the average value of a function $f \in \Omega(M)$ by $A v(f)$.

Let $M=\mathbb{C} P^{n}$ with symplectic form $\omega$ and Hamiltonian action of $G=S^{1}$. Let $\mu$ be the moment map; but we do not assume that $A v(\mu)=0$. Let $\bar{w}$ equal $[\omega+t \mu]_{G}$ in $H_{G}^{2}(M ; \mathbb{R})$. The product structure in $H_{G}^{*}(M ; \mathbb{R})$ is completely determined by expressing $\bar{w}^{n+1}$ in terms of lower powers of $\bar{w}$. Let

$$
\bar{w}^{n+1}=\sum_{i=1}^{n+1} c_{i} \bar{w}^{n+1-i} t^{i}, \quad c_{i} \in \mathbb{R} .
$$

One way to find the $c_{i}$ is the following: for $j \geq 0$,

$$
\bar{w}^{n+1+j}=\sum_{i=1}^{n+1} c_{i} \bar{w}^{n+1+j-i} t^{i} .
$$

So integrating over the fibre $M$ in the Borel construction bundle $M_{G} \rightarrow B G$ gives

$$
\left(\begin{array}{c}
n+1+j \\
1+j
\end{array}\right) t^{1+j} \int_{M} \mu^{1+j} \omega^{n}=\sum_{i=1}^{1+j} c_{i}\left(\begin{array}{c}
n+1+j-i \\
1+j-i
\end{array}\right) t^{1+j} \int_{M} \mu^{1+j-i} \omega^{n}
$$

Thus

$$
\left(\begin{array}{c}
n+1+j \\
1+j
\end{array}\right) A v\left(\mu^{1+j}\right)=\sum_{i=1}^{1+j} c_{i}\left(\begin{array}{c}
n+1+j-i \\
1+j-i
\end{array}\right) A v\left(\mu^{1+j-i}\right)
$$

Since this holds for all $j \geq 0$, one can easily solve for each $c_{i}$ in terms of the average values of the powers of $\mu$. For example, if $j=0$ then $c_{1}=(n+1) A v(\mu)$ and putting $j=1$,

$$
c_{2}=\left(\begin{array}{c}
n+2 \\
2
\end{array}\right) A v\left(\mu^{2}\right)-(n+1)^{2} A v(\mu)^{2} .
$$

Equally, one can solve for each $A v\left(\mu^{j}\right)$ in terms of $c_{1}, \ldots, c_{j}$. This is reasonable because there are other familiar ways to find the $c_{i} s$. Let the fixed point set

$$
M^{G}=\bigcup_{i=1}^{s} F_{i}
$$


where the component $F_{i}$ has dimension $2 r_{i}$. By the equality of Euler characteristics, $\sum_{i=1}^{s}\left(r_{i}+1\right)=n+1$. Let $v_{i}$ be the value of $\mu$ on $F_{i}$, and let $\mu_{j}=v_{i}$ for

$$
\sum_{k=1}^{i-1}\left(r_{k}+1\right)+1 \leq j \leq \sum_{k=1}^{i}\left(r_{k}+1\right) .
$$

So the distinct values of $\mu$ appear with multiplicity, each $v_{i}$ appearing with multiplicity $r_{i}+1$. (If $M^{G}$ is finite, then $s=n+1$, and $\mu_{i}=v_{i}$ for $1 \leq i \leq n+1$.) In terms of these values we have

$$
\prod_{i=1}^{s}\left(\bar{w}-v_{i} t\right)^{r_{i}+1}=\prod_{i=1}^{n+1}\left(\bar{w}-\mu_{i} t\right)=0 .
$$

This follows from the Localization Theorem of Borel, Hsiang and Quillen. For details of this example see [Hsiang 1975, Theorem IV.3] or [Mukherjee 2005, Example 1.3.12]. Thus, for $1 \leq i \leq n+1, c_{i}=(-1)^{i+1} \sigma_{i}$, where $\sigma_{i}$ is the $i$ th elementary symmetric polynomial in $\mu_{1}, \ldots, \mu_{n+1}$.

Now suppose that $M^{G}$ is finite. Thus $s=n+1$ and $r_{i}=0$ for all $i$. Let $U_{i}=\prod_{j \neq i}\left(\bar{w}-\mu_{j} t\right)$. So $U_{i}$ restricts to $\prod_{j \neq i}\left(\mu_{i}-\mu_{j}\right) t^{n}$ at $F_{i}$ and zero at all the other fixed points. Let the equivariant Euler class at $F_{i}$ be $\varepsilon_{i} t^{n}$, normalized so that $\varepsilon_{i}$ is an integer (the product of the weights). Integrating $U_{i}$ over the fibre gives

$$
\int_{M} \omega^{n}=\frac{1}{\varepsilon_{i}} \prod_{j \neq i}\left(\mu_{i}-\mu_{j}\right)
$$

by the integration formula [Atiyah and Bott 1984, 3.8]. (See [Bredon 1972, VIII, Theorem 5.5] (based on the original example of W.-Y. Hsiang), [Petrie 1972] for many related results, or [Mukherjee 2005, Example 1.4.15] for an elementary treatment.)

Meanwhile the Duistermaat-Heckman formula gives

Thus

$$
\int_{M} e^{\mu t} \frac{\omega^{n}}{n !}=\sum_{i=1}^{n+1} \frac{e^{\mu_{i} t}}{\varepsilon_{i} t^{n}}
$$

$$
\left(\begin{array}{c}
n+j \\
j
\end{array}\right) A v\left(\mu^{j}\right)=\sum_{i=1}^{n+1} \frac{\mu_{i}^{n+j}}{\prod_{k \neq i}\left(\mu_{i}-\mu_{k}\right)} .
$$

The last formula is homogeneous in $\mu$, and hence not sensitive to such matters as the parametrization of the circle $(\exp (2 \pi i t)$ or $\exp (i t))$, the sign for $\mu\left(d \mu=i_{V}(\omega)\right.$ or $\left.d \mu=-i_{V}(\omega)\right)$, or the sign for $t\left(d_{G}=d-t i_{V}\right.$ or $\left.d_{G}=d+t i_{V}\right)$. 
The right hand side of Equation (4-2) is a polynomial in $\mu_{1}, \ldots, \mu_{n+1}$, as can be seen from a calculation with Vandermonde determinants or from the fact that each $c_{i}$ is a polynomial in the $\mu_{j}$ 's.

Given a particular linear action, one can use Equation (4-1) to find each $\mu_{i}$, and hence, each $c_{i}$. For example, let $S^{1}$ act on $\mathbb{C} P^{2}$ by $z\left[z_{0}, z_{1}, z_{2}\right]=\left[z_{0}, z^{a} z_{1}, z^{b} z_{2}\right]$, where $a$ and $b$ are integers such that $0<a<b$. Let $\int_{M} \omega^{2}=A$. Choose $\mu$ so that $A v(\mu)=0$. Then one gets

$$
\begin{aligned}
6 A v\left(\mu^{2}\right) & =\frac{1}{3} A\left(a^{2}-a b+b^{2}\right)=c_{2}, \\
10 A v\left(\mu^{3}\right) & =\frac{1}{27} A \sqrt{A}\left(2 a^{3}-3 a^{2} b-3 a b^{2}+2 b^{3}\right)=c_{3} .
\end{aligned}
$$

Thus

$$
\bar{w}^{3}=\frac{1}{3}\left(a^{2}-a b+b^{2}\right) A \bar{w} t^{2}+\frac{1}{27}\left(2 a^{3}-3 a^{2} b-3 a b^{2}+2 b^{3}\right) A \sqrt{A} t^{3} .
$$

(For example, $c_{3}=\mu_{1} \mu_{2} \mu_{3}=-\frac{1}{27} A \sqrt{A}(a+b)(2 a-b)(2 b-a)$.)

Remark 4.1. As is easily seen, any symplectic form on $\mathbb{C} P^{2}$ which is invariant under the linear action above is, up to a nonzero constant multiple, the standard symplectic form plus an invariant exact form. Hence the moment map has the same values at fixed points as does the standard moment map. The latter is

$$
\mu\left(\left[z_{0}, z_{1}, z_{2}\right]\right)=\frac{a\left|z_{1}\right|^{2}+b\left|z_{2}\right|^{2}}{\left|z_{0}\right|^{2}+\left|z_{1}\right|^{2}+\left|z_{2}\right|^{2}}+c,
$$

where $\mathrm{a}$ and $\mathrm{b}$ are as above and $\mathrm{c}$ is any constant. (See [Audin 1991, IV, 4.1.1].) The final formulas are then easy to obtain directly.

\section{Acknowledgments}

We thank the referee for many very helpful suggestions that improved the presentation of this paper. We also thank A. Alekseev and E. Meinrenken for many helpful discussions. An earlier version of this paper was based on the small model, which is shown to be equivalent to the Cartan model in [Alekseev and Meinrenken 2005].

Note added in proof. Some results similar to ours were obtained in [Cairns and Jessup 2004].

\section{References}

[Alekseev and Meinrenken 2005] A. Alekseev and E. Meinrenken, "Equivariant cohomology and the Maurer-Cartan equation", Duke Math. J. 130:3 (2005), 479-521. MR MR2184568 Zbl 1085. 57022

[Allday 2005] C. Allday, "Canonical equivariant extensions using classical Hodge theory", Int. J. Math. Math. Sci. 2005:8 (2005), 1277-1282. MR 2006f:53125 Zbl 02236241 
[Allday and Puppe 1993] C. Allday and V. Puppe, Cohomological methods in transformation groups, Cambridge Studies in Advanced Mathematics 32, Cambridge University Press, Cambridge, 1993. MR 94g:55009 Zbl 0799.55001

[Atiyah and Bott 1984] M. F. Atiyah and R. Bott, "The moment map and equivariant cohomology", Topology 23:1 (1984), 1-28. MR 85e:58041 Zbl 0521.58025

[Audin 1991] M. Audin, The topology of torus actions on symplectic manifolds, Progress in Mathematics 93, Birkhäuser, Basel, 1991. MR 92m:57046 Zbl 0726.57029

[Bredon 1972] G. E. Bredon, Introduction to compact transformation groups, Academic Press, New York, 1972. MR 54 \#1265 Zbl 0246.57017

[Cairns and Jessup 2004] G. Cairns and B. Jessup, "Cohomology operations for Lie algebras", Trans. Amer. Math. Soc. 356:4 (2004), 1569-1583. MR 2005a:17018 Zbl 1049.17020

[Frankel 1959] T. Frankel, "Fixed points and torsion on Kähler manifolds", Ann. of Math. (2) 70 (1959), 1-8. MR 24 \#A1730 Zbl 0088.38002

[Guillemin and Sternberg 1999] V. W. Guillemin and S. Sternberg, Supersymmetry and equivariant de Rham theory, Springer, Berlin, 1999. MR 2001i:53140 Zbl 0934.55007

[Hsiang 1975] W.-Y. Hsiang, Cohomology theory of topological transformation groups, Ergebnisse der Math. und ihrer Grenzgebiete 85, Springer, New York, 1975. MR 54 \#11363

[Kirwan 1984] F. C. Kirwan, Cohomology of quotients in symplectic and algebraic geometry, Mathematical Notes 31, Princeton University Press, Princeton, NJ, 1984. MR 86i:58050

[Lillywhite 2003] S. Lillywhite, "Formality in an equivariant setting", Trans. Amer. Math. Soc. 355:7 (2003), 2771-2793. MR 2004e:55016 Zbl 1021.55006

[McDuff and Salamon 1995] D. McDuff and D. Salamon, Introduction to symplectic topology, Oxford Univ. Press, New York, 1995. MR 97b:58062 Zbl 0844.58029

[Mukherjee 2005] G. Mukherjee (editor), Transformation groups, symplectic torus actions and toric manifolds, edited by G. Mukherjee, Hindustan Book Agency, New Delhi, 2005. MR 2214284

[Petrie 1972] T. Petrie, "Smooth $S^{1}$ actions on homotopy complex projective spaces and related topics”, Bull. Amer. Math. Soc. 78 (1972), 105-153. MR 45 \#6029 Zbl 0247.57010

[Spanier 1966] E. H. Spanier, Algebraic topology, McGraw-Hill Book Co., New York, 1966. MR 35 \#1007

[Warner 1983] F. W. Warner, Foundations of differentiable manifolds and Lie groups, Graduate Texts in Mathematics 94, Springer, New York, 1983. MR 84k:58001 Zbl 0516.58001

Received October 15, 2004. Revised April 29, 2005.

\author{
Christopher AlLDAY \\ DEPARTMENT OF MATHEMATICS \\ UNIVERSITY OF HAWAII \\ HONOLULU, HI 96822 \\ UNITED STATES \\ chris@math.hawaii.edu
}

\title{
VOLKER PUPPE
}

FACHBEREICH FÜR MATHEMATIK UND STATISTIK

UNIVERSITÄT KONSTANZ

KONSTANZ

GERMANY

volker.puppe@uni-konstanz.de 